\title{
Direct observation of interlayer molecular translational motion in a smectic phase and determination of the layer order parameter
}

\author{
Makina Saito, ${ }^{1}$ Jun Yamamoto, ${ }^{2}$ Ryo Masuda, ${ }^{1}$ Masayuki Kurokuzu, ${ }^{1}$ Yohei Onodera, ${ }^{1}$ Yoshitaka Yoda, ${ }^{3}$ and Makoto Seto ${ }^{1}$ \\ ${ }^{1}$ Institute for Integrated Radiation and Nuclear Science, Kyoto University, Kumatori, Osaka 590-0494, Japan \\ ${ }^{2}$ Department of Physics, Graduate School of Science, Kyoto University, Kyoto 606-8224, Japan \\ ${ }^{3}$ Research and Utilization Division, Japan Synchrotron Radiation Research Institute, Sayo, Hyogo 679-5198, Japan
}

(Received 16 April 2019; published 15 August 2019)

\begin{abstract}
The smectic phase shows a layered structure, which is the most fundamental and universal order found in soft materials. However, previous technical limitations made it difficult to fully and microscopically observe the interlayer molecular motions in the smectic phase. We were able to directly measure the interlayer molecular motions by quasielastic scattering spectroscopy using Mössbauer gamma rays for 4 '- $n$-octyl-4-cyanobiphenyl $(8 \mathrm{CB})$. From the measured motions, we evaluated the order parameter of the layer structure and explain the macroscopic diffusion coefficient in the microscopic viewpoint. Our methodology can be applied broadly to determine the layer order parameter of various soft materials.
\end{abstract}

DOI: 10.1103/PhysRevResearch.1.012008

The unique macroscopic property and function of soft materials originate from the coexistence of structural ordering and dynamical motion on a molecular scale [1,2]. Accordingly, understanding the relationship between structure and dynamics on a molecular level provides crucial information on how macroscopic properties can be engineered from their microscopic origins and leads to a rational and efficient design of the macroscopic functionalities. However, thus far, there have been limited attempts to clarify such correlations [3]. In this manuscript, we focus on a thermotropic liquid crystal system that shows a layered smectic $(\mathrm{Sm})$ phase. Layered structures are the most fundamental and universal nanometric order found in soft materials such as lipid and surfactant bilayer systems as well as polymer systems. In the layered structures, anisotropic molecular motions coexist within the layer order and lead to an anisotropic soft response to external forces [4].

Recently, understanding microscopic molecular dynamics in the layered structures has been getting more important for the purposes of revealing the fundamental layer effect on molecular dynamics [5], the caged molecular dynamics in the Sm phase as a model system of supercooled glass formers [6-8], and the mass transportation mechanism in cell and drug delivery systems $[9,10]$. In addition, observation of the interlayer molecular motions allows for direct determination of the layer order parameter in the microscopic layer scale [11], though such direct determination has not been realized yet. So far, diffraction studies determined a translational order parameter in Sm and nematic $(N)$ phases from diffraction intensity by layers; however, the translational order parameter,

Published by the American Physical Society under the terms of the Creative Commons Attribution 4.0 International license. Further distribution of this work must maintain attribution to the author(s) and the published article's title, journal citation, and DOI. which is nonzero in the $N$ phase, is different from the Sm-layer order parameter [12].

Understanding the interlayer molecular motions is also a key to fully understanding the macroscopic diffusion coefficients behavior in the Sm phase. For many Sm phases, anisotropic mobility has been discussed by the macroscopic diffusion coefficients parallel $\left(D_{\|}\right)$and perpendicular $\left(D_{\perp}\right)$ to the layer normal vector obtained by using techniques such as nuclear magnetic resonance (NMR) [13]. The nanometric layer order affects $D_{\|}$, while $D_{\perp}$ is less affected by the layer order and instead shows a liquidlike behavior [11,13,14]. Volino and Dianoux (VD) predicted a general relationship between these macroscopic diffusion coefficients and the layer order parameter using the microscopic periodic potential model [11]. The VD theory can explain the anisotropy of the diffusion coefficients, i.e., $D_{\|}<D_{\perp}$, seen in the Sm phase of many systems via NMR $[11,13,14]$. However, for several liquid crystals, such as 4 - $n$-octyl-4-cyanobiphenyl (8CB), the opposite relation $D_{\|}>D_{\perp}$ exists in the Sm- $A$ phase where the molecular long axis (director) is oriented normal to the layer plane [14]. Thus, the diffusion coefficient behavior in the Sm phase has yet to be explained. So far, quasielastic neutron scattering (QENS) spectroscopy has been used to measure dynamics on a microscopic scale $[11,15,16]$. However, interlayer motions of liquid crystal molecules in the Sm phase are too slow to be fully detected by QENS, and only perpendicular intralayer motions can be fully measured $[13,15,16]$.

Quasielastic Mössbauer gamma-ray scattering (QEGS) spectroscopy using ${ }^{57} \mathrm{Fe}$-nuclear resonance has a $4.7-\mathrm{neV}$ energy resolution, which is about three orders of magnitude higher than the resolution of conventional QENS spectroscopy [17-19]. The validity and uniqueness of QEGS spectroscopy have been previously demonstrated [20]. So far, we showed that QEGS has enough spatial and energy resolution to resolve interlayer molecular translational motions occurring in the 100-ns timescale in the Sm phase [21]. A recent neutron spin echo study for an ionic liquid system with $\mathrm{Sm}-A$ order 
(a)
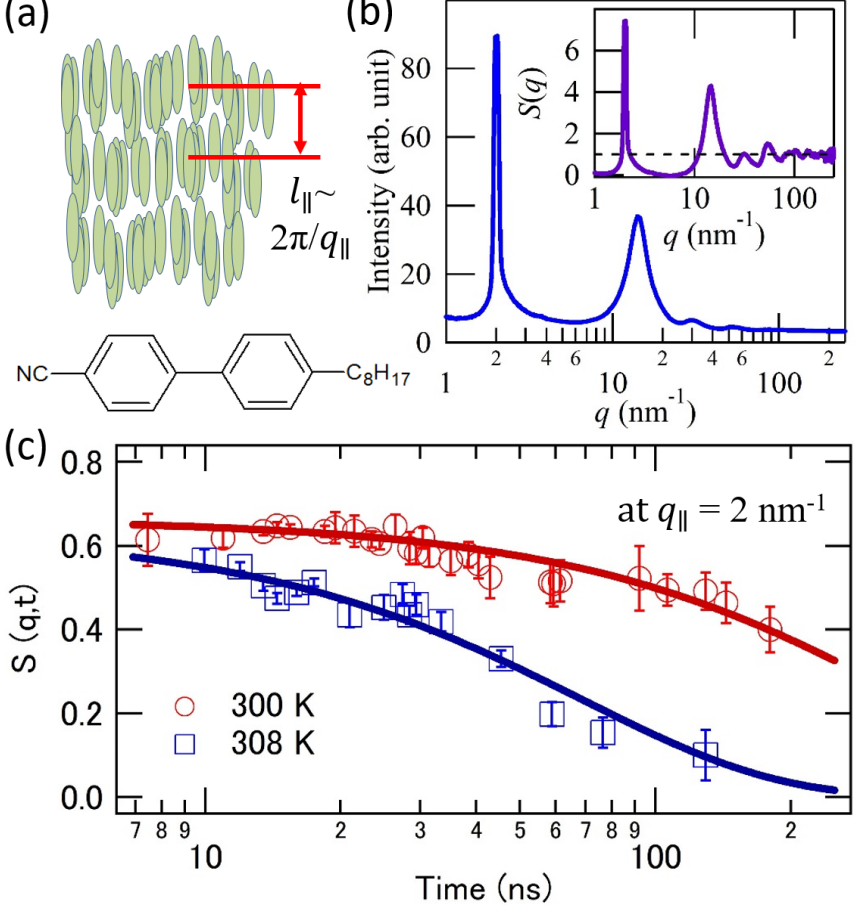

FIG. 1. X-ray diffraction spectrum and normalized intermediate scattering functions obtained by QEGS measurements. (a) Molecular formula of $8 \mathrm{CB}$ and schematic diagram of the molecular arrangement of Sm- $A$ phase. The definition of the interlayer molecular distance $l_{\|}$is shown. (b) Diffraction spectrum of $8 \mathrm{CB}$ obtained at $301 \mathrm{~K}$. The inset shows $S(q)$ obtained by analyzing the diffraction spectrum. (c) Examples of the normalized intermediate scattering function $S(q, t)$ obtained by QEGS measurements at $q_{\|} \sim 2.0 \mathrm{~nm}^{-1}$ at $300 \mathrm{~K}$ (circles) and at $308 \mathrm{~K}$ (squares) and the corresponding fitting curves.

suggested a similar timescale for the interlayer motion [22]. In this study, we measure interlayer molecular translational motions in the $\mathrm{Sm}-A$ phase of $8 \mathrm{CB}$ with varying temperature ( $T$ ) toward the Sm- $A-N$ transition. Using the microscopic periodic potential model, we obtain the layer order parameter from interlayer molecular translational motions. In addition, we attempt to explain the behavior of the macroscopic diffusion coefficient obtained by NMR based on the microscopic dynamical picture obtained by QEGS.

The molecular formula of $8 \mathrm{CB}$ is shown in Fig. 1(a). We purchased 8CB (with purity $>97 \%$ ) from Wako pure chemical industries (Osaka, Japan) and used it in the experiment without further purification. The phase transitions of $8 \mathrm{CB}$ follow the phase sequence $\mathrm{Sm}-A-306 \mathrm{~K}-N-314 \mathrm{~K}$-liquid isotropic $(I)$ phase. X-ray diffraction study in the $q$ region of $1-5 \mathrm{~nm}^{-1}$ was performed by using $\mathrm{Cu} K \alpha \mathrm{x}$ rays in a Nanopix (Rigaku, Tokyo, Japan), where $q$ is the magnitude of the wave vector transfer defined as $q=4 \pi / \lambda \sin (\theta / 2)$ with $\lambda$ being the wavelength of the incident $\mathrm{x}$ ray and $\theta$ being the scattering angle. In addition, diffraction study in the $q$ region of $3-257 \mathrm{~nm}^{-1}$ was performed at the high-energy x-ray diffraction beamline (BL04B2) of SPring-8 in Japan [23]. The diffraction spectrum obtained at $301 \mathrm{~K}$ is shown in Fig. 1(b). The peaks located at $q_{\|} \sim 2.0 \mathrm{~nm}^{-1}$ and $q_{\perp} \sim 15 \mathrm{~nm}^{-1}$ reflect the distance between molecules in the interlayer and intralayer directions, respectively. Here, $q_{\|} \sim 2.0 \mathrm{~nm}^{-1}$ is associated with the interlayer molecular distance $l_{\|}=2 \pi / q_{\|} \sim 3 \mathrm{~nm}$. Figure 1(a) shows the schematic diagram of the molecular arrangement of the $\mathrm{Sm}-A$ phase. Although the $\mathrm{Sm}$ phase of $8 \mathrm{CB}$ is known to have a partial bilayer structure [24,25], the details of the layer structure are unrelated to this study. Following the definition of Faber and Ziman, we evaluated the static structure factor $S(q)$ from the diffraction spectrum $I(q)$ obtained at $301 \mathrm{~K}$, as shown in the inset of Fig. 1(b) [26].

QEGS experiments were performed at the nuclear resonant scattering beamline (BL09XU) of SPring-8 (Japan) in an operating mode with a bunch interval of $684.3 \mathrm{~ns}$. Directional Mössbauer gamma rays (with an energy of $14.4 \mathrm{keV}$ and an energy width of $\sim 4.7 \mathrm{neV}$ ) from the ${ }^{57} \mathrm{Fe}$ nucleus were utilized for the QEGS measurements using multiline time domain interferometry [19]. We applied a magnetic field (MF) $(\sim 1.4 \mathrm{kG})$ to align the director of the $8 \mathrm{CB}$ molecules using permanent magnets and to suppress the molecular rotation and accompanying layer undulation. We first applied the MF to $8 \mathrm{CB}$ in the $I$ phase at well above the $I-N$ transition temperature of $314 \mathrm{~K}$. Then, the sample was cooled down to the liquid crystal phase under constant MF. We confirmed that the azimuthal angle distribution of the small-angle scattering from the interlayer molecular correlation of $8 \mathrm{CB}$ molecules is sufficiently narrow at $5.5^{\circ}$ around its average direction. We placed the detector to cover the whole azimuthal angle distribution of the small angle scattering. The $T$-dependent QEGS measurement was performed on heating at $q_{\|} \sim 2.0 \mathrm{~nm}^{-1}$ with a $q$ resolution of $0.9 \mathrm{~nm}^{-1}$. The $q$ resolution was selected to include whole small-angle diffraction peaks at all measurement temperatures.

In Fig. 1(c), we show examples of the intermediate scattering function [ISF; $S(q, t)$ ] normalized by the static structure factor obtained at $300 \mathrm{~K}$ (circles) and $308 \mathrm{~K}$ (squares) at $q_{\|}$. See Ref. [19] for details of the data analysis. The spectra contain direct information of the molecular translational motion in the interlayer scale. Because, at the microscopic level, the molecular alignment in the Sm- $A$ phase of $8 \mathrm{CB}$ is far from a perfect periodic structure [25], the interlayer jump distance is expected to be rather distributed. In cases with enough distribution, the relaxation of ISF can be expressed by an exponential function [27]. We assumed an exponential function, $f \exp \left(-t / \tau_{\|}\right)$, for the relaxation behavior of $S(q, t)$, where $t, \tau_{\|}$, and $f$ represent time, the interlayer molecular translational relaxation time, and the factor for the strength of the relaxation, respectively $[21,28]$. The relaxation curves obtained by fitting with the exponential function are shown as the solid lines in Fig. 1(c). The fitting could be successfully performed for all spectra measured between 300 and $308 \mathrm{~K}$ suggesting that the obtained relaxation time $\tau_{\|}$nicely reflects the timescale of the interlayer molecular motion. In the Sm system, ISF is often expressed by the stretched exponential form [7]. The present result suggests that the stretching degree is too small to be detected in the 8CB case because of its relatively lower layer order. The obtained $T$ dependence of $\tau_{\|}$is shown in Fig. 2(a). We confirmed that the interlayer molecular translational motion occurs in the timescale of $100 \mathrm{~ns}$ in the Sm phase of $8 \mathrm{CB}$, as expected from a previous simulation study [24]. In the $\mathrm{Sm}-A$ phase, it was found that the $T$ dependence of $\tau_{\|}$cannot be described by the Arrhenius law. Instead, $\tau_{\|}$rapidly decreases toward the $S \mathrm{~m}-A-N$ phase 


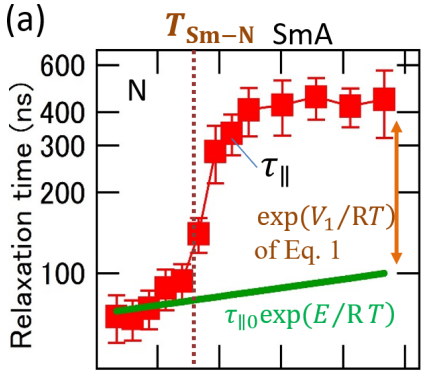

3.263 .283 .303 .323 .34 $1000 / \mathrm{T}\left(\mathrm{K}^{-\mathrm{P}}\right)$

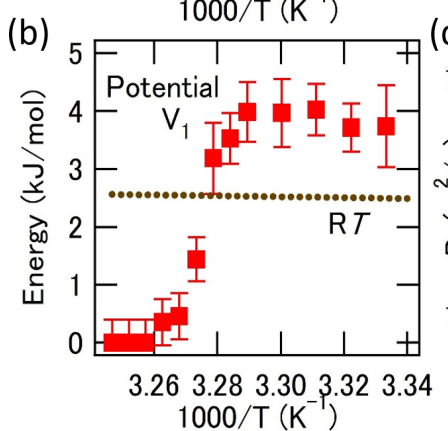
$\left.1000 / \mathrm{T}^{-1}\right)$ (c)

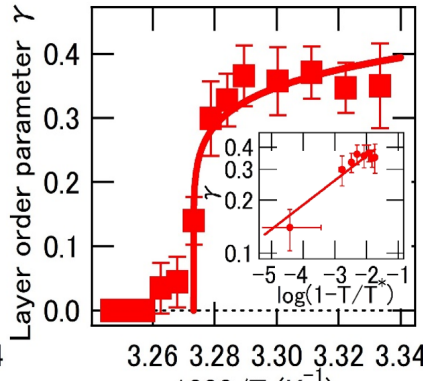

(d)

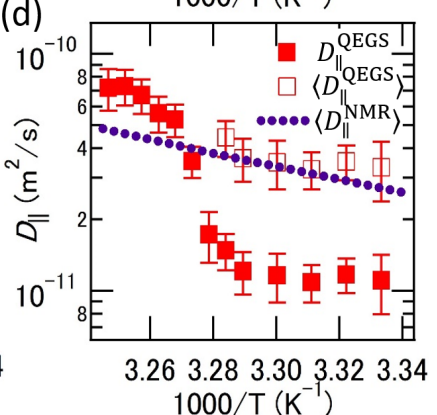

FIG. 2. $T$ dependence of the interlayer relaxation time $\tau_{\|}$and the evaluated layer potential, layer order parameter, and interlayer diffusion coefficient. (a) $T$ dependence of the relaxation time $\tau_{\|}$ obtained at $q_{\|}=2.0 \mathrm{~nm}^{-1}$ (red squares). The solid line represents the factor $\tau_{\| 0} \exp (E / R T)$ of Eq. (1). The dotted line represents the Sm- $A-$ $N$ phase transition temperature. (b) $T$ dependence of the potential $V_{1}$ obtained from $\tau_{\|}$using the microscopic periodic potential model. The dotted line represents the $R T$ curve. (c) $T$ dependence of the order parameter $\gamma$ evaluated from $V_{1}$. The solid line represents the fitting curve by assuming a critical behavior. Inset shows the $\gamma$ dependence on $1-T / T^{*}$. (d) $T$ dependence of the interlayer diffusion coefficient $D_{\|}^{\text {QEGS }}$ directly obtained from $\tau_{\|}$using the jump diffusion model (filled squares). The dotted line represents the diffusion coefficient behavior $\left\langle D_{\|}^{\mathrm{NMR}}\right\rangle$ obtained by NMR [14]. The empty squares represent the averaged diffusion coefficient $\left\langle D_{\|}^{\text {QEGS }}\right\rangle$ evaluated by using both the $q$ and $T$ dependence studies considering the dynamical inhomogeneity.

transition temperature $T_{\mathrm{Sm}-N}$. Thus, we could directly measure the molecular motion in the interlayer scale of the $\mathrm{Sm}$ phase and observe the $T$-dependent behavior toward $T_{\mathrm{Sm}-N}$.

We next aim to find an expression for the relaxation time of the microscopic interlayer translational motion. The periodic potential due to the layered order can be expressed as $V(\mathrm{z})=$ $-V_{1} / 2 \cos \left(z q_{\|}\right)$, where $z$ is the coordinate of the layer normal direction and $V_{1}$ is the height of the potential [11]. Here, $V_{1}$ is closely related to the layer order parameter. The VD theory treats the macroscopic diffusion coefficient at spatial scales much larger than the interlayer distance using the periodic potential model [11]. Considering the microscopic molecular motion under the potential barrier, the interlayer molecular relaxation time $\tau_{\|}$is obtained as

$$
\tau_{\|}(T)=\tau_{\| 0} \exp (E / R T) \exp \left(V_{1} / R T\right),
$$

where $R$ is the gas constant, $E$ is the intrinsic activation energy in the absence of a periodic potential, and $\tau_{\| 0}$ is the frequency factor. The factor $\tau_{\| 0} \exp (E / R T)$ represents the timescale of the attempt to traverse the potential barrier at each temperature. This is the microscopic representation of the VD theory $[11,29,30]$.

First, we determine the factor $\tau_{\| 0} \exp (E / R T)$ to obtain the periodic potential $V_{1}$ from $\tau_{\|}$. To achieve this, we measured the relaxation times in the $N$ phase close to $T_{\mathrm{Sm}-N}$, where $V_{1}$ can be assumed to be 0 . By fitting the relaxation times in the $N$ phase using the $E$ value of $31 \mathrm{~kJ} / \mathrm{mol}$ determined by previous NMR experiments, we obtained $\tau_{\| 0}=0.37 \mathrm{ps}$ [14]. The obtained $\tau_{\| 0} \exp (E / R T)$ factor of Eq. (1) is shown as the solid line in Fig. 2(a). The difference between the experimentally measured relaxation time (red squares) and the factor $\tau_{\| 0} \exp (E / R T)$ is due to the periodic potential $\exp \left(V_{1} / R T\right)$ from Eq. (1). We determined $V_{1}$ from the measured relaxation time; the obtained $V_{1}$ values are shown as the filled squares in Fig. 2(b). We found that the obtained periodic potential $V_{1}$ is $\sim 4 \mathrm{~kJ} / \mathrm{mol}$ at room temperature $(300 \mathrm{~K})$ and rapidly decreases as the temperature approaches $\sim T_{\mathrm{Sm}-N}$.

We next obtain the order parameter from $V_{1}$. The potential $V_{1}$ is related to the layer order parameter $\gamma$ given by $\gamma=$ $\mathrm{I}_{1}\left(V_{1} / 2 R T\right) / \mathrm{I}_{0}\left(V_{1} / 2 R T\right)$, where $\mathrm{I}_{n}$ is the $n$th order modified Bessel function of the first kind [11]. The obtained layer order parameters are shown as the filled squares in Fig. 2(c). The $T$ dependence of the order parameter is often expressed to follow a critical behavior $\gamma(T) \propto\left(1-T / T^{*}\right)^{\beta}$, where $\beta$ is the critical index and $T^{*}$ is the critical temperature; hence, we use this expression to obtain the fitting curve shown as the solid line in Fig. $2(\mathrm{c})$ where $T^{*} \sim 306 \mathrm{~K}\left(\sim T_{\mathrm{Sm}-N}\right)$ with a sub-K accuracy and $\beta=0.14( \pm 0.04)$. The observed behavior is consistent with the second-order or very weak first-order transition nature of the $\mathrm{Sm}-A-N$ phase transition of $8 \mathrm{CB}$ [31]. Accordingly, the layer order parameter could be directly obtained from the interlayer molecular motion. Our methodology can be applied broadly to many layered systems including $\mathrm{Sm}$ systems with high-order structure and Sm films [4].

We then estimate the diffusion coefficient from the measured interlayer relaxation time to understand the macroscopic diffusion coefficient by NMR. When the periodic potential barrier is high enough such that $V_{1} \gg R T$, molecular motions are treated as jump motions $[11,29,30]$. In Fig. 2(b), the $R T$ curve is shown for comparison. Between 300 and $305 \mathrm{~K}$, we assume that $V_{1} \gg R T$ and evaluate the interlayer diffusion coefficient $D_{\|}$using the one-dimensional jump diffusion model such that $D_{\|}^{\mathrm{QEGS}}=l_{\|}^{2} /\left(2 \tau_{\|}\right)$, where $l_{\|}$is the average interlayer distance corresponding to the step length of the interlayer motion and $\tau_{\|}$is the time required for the step. The $D_{\|}^{\text {QEGS }}$ values obtained by QEGS are shown as the filled squares in Fig. 2(d). The diffusion coefficient obtained by NMR is also shown as the dotted line in Fig. 2(d) [14]. The figure shows that the slopes of the diffusion coefficients are similar; however, the $D_{\|}^{\mathrm{QEGS}}$ values obtained by QEGS are approximately three times smaller than those by NMR.

The difference in the diffusion coefficients obtained by QEGS and NMR can be explained by the difference in the objects they measure. The NMR measures self-diffusion motions in the spatial scale of the micrometer [14]. On the other hand, QEGS selectively observes molecular motions in the interlayer scale of the nanometer. In the Sm phase, intrinsic layer order fluctuations always exist due to the Landau-Peierls 
(a) More contribute to $S(q)$ peak (b)

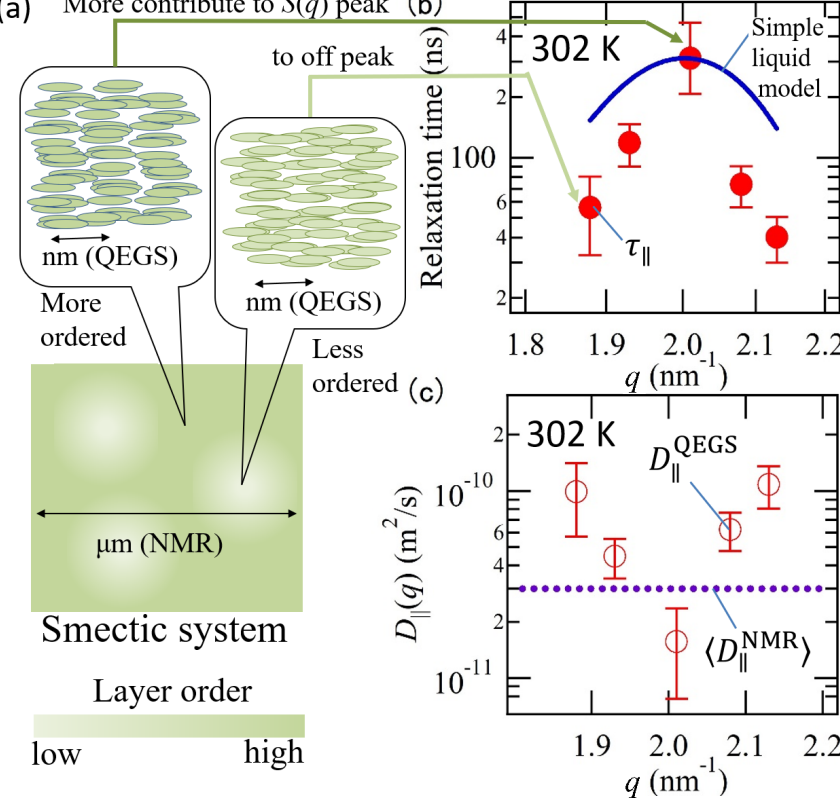

FIG. 3. $q$ dependence of the relaxation time obtained at $302 \mathrm{~K}$ around $q_{\|} \sim 2.0 \mathrm{~nm}^{-1}$ and the evaluated interlayer diffusion coefficient. (a) Schematic picture of dynamical inhomogeneity due to the LPI and defects in the Sm system and objects measured by NMR and QEGS. (b) $q$ dependence of the relaxation time obtained at $302 \mathrm{~K}$ around $q_{\|} \sim 2.0 \mathrm{~nm}^{-1}$. The solid line represents the simple liquid model assuming dGN narrowing, $\tau_{\mathrm{dGN}}(q)$. The liquid model cannot explain the experimental result, which suggests that dynamical inhomogeneity is present. (c) $q$ dependence of the diffusion coefficient evaluated by assuming the jump diffusion model. The dotted line represents the diffusion coefficient obtained by NMR [14].

instability (LPI) [32]. In addition, inhomogeneity of the layer order is caused by defects, where molecular motions are faster than these in more ordered regions [33,34]. The diffusion coefficient obtained by NMR is treated as the averaged diffusion coefficient over regions with structural and dynamical inhomogeneity; therefore, we write it as $\left\langle D_{\|}^{\mathrm{NMR}}\right\rangle$. On the other hand, because QEGS measures the coherent quasielastic scattering, it selectively observes nanometric molecular motions in regions with a more-ordered layer because they have a higher (quasi-)elastic scattering intensity. In addition, a more-ordered layer has slower molecular motions than average motions. This explains why the diffusion coefficient $D_{\|}^{\text {QEGS }}$ obtained by QEGS is smaller than $\left\langle D_{\|}^{\mathrm{NMR}}\right\rangle$, as shown in Fig. 2(d).

To better explain the $\left\langle D_{\|}^{\mathrm{NMR}}\right\rangle$ value qualitatively from the microscopic viewpoint, we evaluate the average diffusion coefficient by QEGS. The effect of the dynamical inhomogeneity due to the LPI is demonstrated by the broadening of the small-angle scattering peak in the x-ray diffraction spectrum [35], where the off-center scattering originates from regions with a less-ordered layer. Similarly, we assume defect regions with lower layer order also contribute to the off-center scattering. In Fig. 3(a), we show the schematic picture of objects measured by NMR and QEGS and show how molecular motions in regions with different layer orders are selectively observed by the $q$-dependent measurement of QEGS. This shows that the average diffusion coefficient $\left\langle D_{\|}^{\mathrm{QEGS}}\right\rangle$ can be evaluated by averaging the $D_{\|}^{\text {QEGS }}$ obtained by QEGS at different $q$ positions. Therefore, we studied the $q$ dependence of the relaxation time around the small-angle scattering peak at several $q$ points between 1.88 and $2.13 \mathrm{~nm}^{-1}$ with a higher $q$ resolution of $0.09 \mathrm{~nm}^{-1}$ under the MF of $\sim 1.4 \mathrm{kG}$ at $302 \mathrm{~K}$. The $q$ dependence of the relaxation time at $302 \mathrm{~K}$ in the $\mathrm{Sm}-A$ phase, as shown in Fig. 3(b), has a maximum at $q_{\|} \sim$ $2.0 \mathrm{~nm}^{-1}$. We found that molecular motions in regions with a less-ordered layer are much faster than those in regions with a more-ordered layer.

We next consider if the behavior cannot be explained by the liquidlike $q$ dependence known as de Gennes narrowing (dGN). The relaxation time of a coherent ISF is expressed as $\tau_{\mathrm{dGN}}(q)=S(q) \tau^{\text {self }}(q)$, where $\tau^{\text {self }}(q) \propto q^{-2}$ is the relaxation time of a self-ISF obtained by incoherent QENS [36-38]. The $\tau_{\mathrm{dGN}}(q)$ determined from the measured $S(q)$ considering the $q$ resolution of the measurement is shown as the solid curve in Fig. 3(b). The $\tau_{0}^{\text {self }}$ parameter was taken as the experimental $\tau_{\|}$at the center for the small-angle scattering of $q=2.0 \mathrm{~nm}^{-1}$. Figure 3(b) reveals that the measured relaxation time off the peak center, where we observed regions with a less-ordered layer, is approximately several times faster than the predicted $\tau_{\mathrm{dGN}}(q)$. The observed difference is large compared to the differences seen in previous results on simple liquids $[37,38]$ and more complex system [39]. Therefore, the $q$-dependent result may not be explained solely by dGN suggesting the presence of dynamical inhomogeneity due to the LPI and defects.

We evaluate the average diffusion coefficient $\left\langle D_{\|}^{\mathrm{QEGS}}\right\rangle$ from the $q$-dependent study and compare it with $\left\langle D_{\|}^{\mathrm{NMR}}\right\rangle$. In Fig. 3(c), the $q$ dependence of $D_{\|}^{\text {QEGS }}$ calculated from the relaxation time assuming the jump diffusion model is shown. The observed $q$ dependence has a minimum in $D_{\|}^{\text {QEGS }}$ at the small-angle scattering peak below the value determined by NMR (dotted line) [14]. However, the $D_{\|}^{\text {QEGS }}$ obtained at the off-center $q$ region is larger than the value by NMR. This is consistent with the picture that $\left\langle D_{\|}^{\mathrm{NMR}}\right\rangle$ is the averaged diffusion coefficient over regions with different layer orders. We roughly evaluate the averaged relaxation times $\left\langle D_{\|}^{\mathrm{QEGS}}\right\rangle$ from the $q$-dependent $D_{\|}^{\mathrm{QEGS}}$ obtained by QEGS as $\left\langle D_{\|}^{\mathrm{QEGS}}\right\rangle=$ $\sum_{i=1}^{5} I\left(q_{i}\right) W_{D_{\|}}\left(q_{i}\right) D_{\|}^{\mathrm{QEGS}}\left(q_{i}\right) / \sum_{i=1}^{5} I\left(q_{i}\right) W_{D_{\|}}\left(q_{i}\right)$, where $q_{i}$ is the $q$ position of each measurement and $I$ and $W_{D_{\|}}$are the scattering intensity and the statistical weight of each $D_{\|}^{\text {QEGS }}$, respectively. The obtained $\left\langle D_{\|}^{\mathrm{QEGS}}\right\rangle=3 \times 10^{-11} \mathrm{~m}^{2} / \mathrm{s}$ is three times larger than $D_{\|}^{\text {QEGS }}$ directly obtained by QEGS at the small-angle diffraction peak. Despite only having five points to do the estimation, our results follow $\left\langle D_{\|}^{\text {QEGS }}\right\rangle \sim\left\langle D_{\|}^{\text {NMR }}\right\rangle \sim$ $3 \times 10^{-11} \mathrm{~m}^{2} / \mathrm{s}$ at $302 \mathrm{~K}$. Between 300 and $304.5 \mathrm{~K}$, the order parameters have similar values of around 0.35 (ranging between 0.33 and 0.37), as shown in Fig. 2(c), suggesting similarities in the degree of the dynamical inhomogeneity in the $T$ region. Therefore, in the $T$ region, $\left\langle D_{\|}^{\mathrm{QEGS}}\right\rangle$ can be evaluated from $D_{\|}^{\text {QEGS }}$ by taking into account the factor of 3 in the first approximation. The evaluated $\left\langle D_{\|}^{\text {QEGS }}\right\rangle$ is plotted in Fig. 2(d), confirming that $\left\langle D_{\|}^{\mathrm{QEGS}}\right\rangle$ agrees well with $\left\langle D_{\|}^{\mathrm{NMR}}\right\rangle$. 
We conclude that the macroscopic diffusion coefficient could be explained by the microscopic interlayer molecular translational relaxation time by considering the intrinsic dynamical inhomogeneity. We showed that the diffusion coefficient obtained by NMR is not directly affected by the layer order parameter but is instead largely affected by other additional factors such as intrinsic dynamical inhomogeneity due to the LPI and defects. This complex nature of the macroscopic diffusion coefficient is the origin of its anomalous behavior reported previously [14]. We demonstrated the importance of understanding the microscopic dynamics to fully elucidate the origin of the macroscopic transport property.

The authors thank Professor S. Kishimoto [High Energy Accelerator Research Organization (KEK)] for his development of the avalanche photodiode detectors. We also thank the accelerator group of SPring-8 for their support. These experiments were performed in SPring- 8 with the approval of the Japan Synchrotron Radiation Research Institute (JASRI) (Proposal No. 2015A1224, No. 2017A1096, and No. 2017A0134). The small-angle $\mathrm{x}$-ray diffraction experiment using Nanopix was performed with a support of Dr. R. Inoue. We are grateful to Dr. K. Ohara (JASRI) and Dr. S. Kohara [National Institute for Materials Science (NIMS)] for their support for the $\mathrm{x}$ ray diffraction experiment at BL04B2 in SPring-8. We are grateful to Dr. E. G. Kelley [National Institute of Standards and Technology (NIST)] and Dr. M. Nagao (NIST) for their discussion and critical reading of the manuscript. This work was supported by a Japan Society for the Promotion of Science (JSPS) KAKENHI Grant-in-Aid for Scientific Research (S) (Grant No. JP24221005) and Grant-in-Aid for Young Scientists (B) (Grant No. JP15K17736), and by JST CREST (Grant No. JPMJCR1424), Japan.
[1] I. W. Hamley, Introduction to Soft Matter: Polymers, Colloids, Amphiphiles and Liquid Crystals (Wiley, West Sussex, UK, 2000).

[2] P. M. Chaikin and T. C. Lubensky, Principles of Condensed Matter Physics (Cambridge University Press, Cambridge, 2000).

[3] T. Yamaguchi, M. Saito, K. Yoshida, T. Yamaguchi, Y. Yoda, and M. Seto, J. Phys. Chem. Lett. 9, 298 (2018).

[4] P. Oswald and P. Pieranski, Smectic and Columnar Liquid Crystals: Concepts and Physical Properties Illustrated by Experiments (Taylor \& Francis, Boca Raton, FL, 2005).

[5] M. P. Lettinga and E. Grelet, Phys. Rev. Lett. 99, 197802 (2007).

[6] M. Bier, R. van Roij, M. Dijkstra, and P. van der Schoot, Phys. Rev. Lett. 101, 215901 (2008).

[7] A. Patti, D. El Masri, R. van Roij, and M. Dijkstra, Phys. Rev. Lett. 103, 248304 (2009).

[8] R. Ni, S. Belli, R. van Roij, and M. Dijkstra, Phys. Rev. Lett. 105, 088302 (2010).

[9] Z. H. Nguyen, M. Atkinson, C. S. Park, J. Maclennan, M. Glaser, and N. Clark, Phys. Rev. Lett. 105, 268304 (2010).

[10] L. Alvarez, M. P. Lettinga, and E. Grelet, Phys. Rev. Lett. 118, 178002 (2017).

[11] F. Volino and A. J. Dianoux, Mol. Phys. 36, 389 (1978).

[12] G. G. Alexander, S. M. King, R. M. Richardson, and H. Zimmermann, Liq. Cryst. 37, 961 (2010).

[13] G. J. Krüger, Phys. Rep. 82, 229 (1982).

[14] S. V. Dvinskikh, I. Furó, H. Zimmermann, and A. Maliniak, Phys. Rev. E 65, 061701 (2002).

[15] R. D. Lefort, D. Morineau, R. Guégan, C. Ecolivet, M. Guendouz, J.-M. Zanotti, and B. Frick, Phys. Chem. Chem. Phys. 10, 2993 (2008).

[16] A. J. Dianoux, A. Heidemann, F. Volino, and H. Hervet, Mol. Phys. 32, 1521 (1976).

[17] A. Q. R. Baron, H. Franz, A. Meyer, R. Rüffer, A. I. Chumakov, E. Burkel, and W. Petry, Phys. Rev. Lett. 79, 2823 (1997).
[18] G. V. Smirnov, V. G. Kohn, and W. Petry, Phys. Rev. B 63, 144303 (2001).

[19] M. Saito, R. Masuda, Y. Yoda, and M. Seto, Sci. Rep. 7, 12558 (2017).

[20] M. Saito, S. Kitao, Y. Kobayashi, M. Kurokuzu, Y. Yoda, and M. Seto, Phys. Rev. Lett. 109, 115705 (2012).

[21] M. Saito, M. Seto, S. Kitao, Y. Kobayashi, M. Kurokuzu, J. Yamamoto, and Y. Yoda, J. Phys. Soc. Jpn. 81, 023001 (2012).

[22] F. Nemoto, M. Kofu, M. Nagao, K. Ohishi, S. Takata, J. Suzuki, T. Yamada, K. Shibata, T. Ueki, Y. Kitazawa, M. Watanabe, and O. Yamamuro, J. Chem. Phys. 149, 054502 (2018).

[23] S. Kohara, M. Itou, K. Suzuya, Y. Inamura, Y. Sakurai, Y. Ohishi, and M. Takata, J. Phys.: Condens. Matter 19, 506101 (2007).

[24] Y. Lansac, M. A. Glaser, and N. A. Clark, Phys. Rev. E 64 051703 (2001).

[25] M. F. Palermo, A. Pizzirusso, and C. Zannoni, J. Chem. Phys. 138, 204901 (2013).

[26] T. E. Faber and J. M. Ziman, Philos. Mag. 11, 153 (1965).

[27] C. T. Chudley and R. J. Elliott, Proc. Phys. Soc. 77, 353 (1961).

[28] We found the relaxation times obtained with MF of 1.4 and $6 \mathrm{kG}$ agree with each other within the measurement error suggesting that the MF does not affect the relaxation time of the translational diffusion as expected by comparison of the energy scale of the interaction with the kinetic energy.

[29] R. M. Richardson, A. J. Leadbetter, D. H. Bonsor, and G. J. Krüger, Mol. Phys. 40, 741 (1980).

[30] R. M. Richardson, A. J. Leadbetter, and C. Frost, Mol. Phys. 45, 1163 (1982).

[31] H. Yoshida, Phys. Lett. A 172, 267 (1993).

[32] L. D. Landau and E. M. Lifshitz, Statistical Physics (Pergamon Press, Oxford, 1980).

[33] R. L. Blumberg Selinger, Phys. Rev. E 65, 051702 (2002).

[34] We note that, in the case of screw dislocations, helically connected layers allow for interlayer molecular diffusion by intralayer diffusion process around the dislocation core; this can be the other interlayer diffusion mechanism. However, in usual 
thermotropic Sm systems whose $D_{\|}$is the same order of $D_{\perp}$ (as $8 \mathrm{CB})$, the mechanism shows negligible effect [33].

[35] J. Als-Nielsen, J. D. Litster, R. J. Birgeneau, M. Kaplan, C. R. Safinya, A. Lindegaard-Andersen, and S. Mathiesen, Phys. Rev. B 22, 312 (1980).
[36] P. G. de Gennes, Physica (Amsterdam, Neth.) 25, 825 (1959). [37] K. Sköld, Phys. Rev. Lett. 19, 1023 (1967).

[38] K. N. Pathak and K. S. Singwi, Phys. Rev. A 2, 2427 (1970).

[39] L. Hong, N. Smolin, and J. C. Smith, Phys. Rev. Lett. 112, 158102 (2014). 